\title{
FOURTEEN CASES OF IDIOPATHIC MYOCARDITIS IN INFANTS AND CHILDREN
}

\author{
BY \\ HOWARD WILLIAMS, R. N. O'REILLY and ALAN WILLIAMS \\ From the Departments of Clinical Research and Pathology, The Children's Hospital, Melbourne
}

(RECEIVED FOR PUBLICATION MARCH 16, 1953)

The purpose of this paper is to present 14 case histories of infants and children with 'idiopathic' myocarditis in order to clarify the clinical and pathological features of the disease, to show how a diagnosis can be established, and to point out some common errors in diagnosis.

Idiopathic myocarditis was first described in 1899 by Fiedler in an adult patient, and during the next 30 years isolated reports of affected children and adults were recorded, mainly in the continental literature. Following a paper by Scott and Saphir in 1929 in which they reviewed the literature of 30 cases and reported two additional adult patients, a number of case reports in infants and children appeared in the American literature (Kenny and Sanes, 1933; Maslow and Lederer, 1933; Smith and Stephens, 1938; Greenebaum, Felson and Zeligs, 1941). In 1944, Saphir, Wile and Reingold reviewed all types of myocarditis in children and recorded the clinical and post-mortem data of 97 patients, three of whom had idiopathic myocarditis. The conclusions from this review were that there was little known clinically about idiopathic myocarditis which was usually unsuspected during life and not infrequently caused sudden death. The clinical findings, such as were available, suggested that the outstanding signs and symptoms were tachycardia out of all proportion to temperature, cyanosis and cardiac enlargement. The few patients who had electrocardiographic tracings showed flat, isoelectric or inverted $T$ waves in the three standard leads. The morbid anatomical changes were an enlarged heart, often with hypertrophy, and an interstitial inflammatory reaction in the myocardium which frequently showed toxic and degenerative changes. There was rarely any evidence of pericarditis or endocarditis. Saphir et al. further stated that the aetiology was unknown, and clinically and pathologically the lesion was not associated with any known infection. Bacteriological examination of the heart and other tissues gave negative results, and pathological lesions were not detected in other organs. However, the pathological lesions in the myocardium were identical with those seen in some of the infectious diseases. An allergic basis and also an association with status thymicolymphaticus had been postulated by a few workers, but the evidence for this was inadequate. Clinically and pathologically the disease in no way resembled rheumatic infection.

Over the past eight years reports of a series of six cases by Keller in 1945, and of five cases by Lind and Hultquist in 1949 have helped to establish a better clinical picture of the disease. Keller described the features of rapid distressed breathing and cyanosis, tachycardia out of all proportion to the temperature, an enlarged liver, a grossly enlarged heart radiologically, and changes in the $T$ waves in the electrocardiogram. Four of the five patients reported by Lind and Hultquist were under the age of 1 month and three of them had paroxysmal tachycardia.

\section{Clinical and Pathological Material and Methods}

Over the past three years, August, 1949, to December, 1952, 14 patients with idiopathic myocarditis have been studied, 12 of them at the Children's Hospital, Melbourne, and two in the Hamilton Base Hospital, Victoria. All patients were carefully observed clinically and electrocardiographic and radiological studies of the heart were made in most. In those patients who died routine macroscopic and microscopic examination was made of all organs, and histological study of the heart included sections from both ventricles, auricles, the interventricular septum and valves.

\section{Classification}

Classification serves as a basis for presentation of the patients rather than as a means of separating them into definite groups with distinctive clinical and 
pathological phenomena. Clinically every variant was seen from severe acute cardiac failure, which was frequently rapidly fatal, to less severe, more insidious subacute failure.

The patients will be discussed in two groups. 1. The acute type was characterized clinically by the sudden onset of severe cardiac failure. The pathological changes in the seven patients who died consisted of widespread myocardial damage and interstitial cellular inflammation. The two surviving patients recovered completely. 2. The subacute and chronic type was characterized clinically by a rather longer history and more gradual development of cardiac failure. The pathological changes in the one patient who died were essentially the same as those in the acute group. The four surviving patients all had some permanent cardiac damage.

\section{The Acute Type}

There were nine patients in this group, the ages ranging from 12 days to 5 years. The initial manifestations of the malady were commonly mild, the mother attributing them to a minor ailment. Some had slight fever and were irritable and difficult to manage, others were vaguely not well, while some refused their food, vomited or had loose stools. One had a sore throat, another a "cold". None of the patients were in contact with infectious diseases and the parents and other members of the family were well at the time the child became ill. These symptoms were present for a few days or a week before the onset of cardiac failure, when the child rapidly became seriously ill. Circulatory and respiratory embarrassment were the outstanding clinical features. Pallor was a striking feature and varying degrees of cyanosis were noted in all patients. Some were restless and had a rather apprehensive look, while others were apathetic. The skin was cold, frequently mottled, often clammy and occasionally wet, and the temperature was either subnormal, normal or slightly elevated. The respirations were rapid, often as high as 90 to 100 per minute, usually deep with a short expiratory grunt or slight wheeze. The chest was frequently barrel shaped from pulmonary emphysema. The percussion note over the entire chest was hyper-resonant and at times the upper margin of liver dullness was displaced downwards owing to the low position of the diaphragm. The breath sounds were harsh and in some patients crepitations were heard at the lung bases. The pulse rate was rapid, usually between 150 and 170 per minute, weak and thready, and the blood pressure low. Cardiac enlargement was always present but often could not be detected clinically as the rapid respira- tory rate and restlessness of the infant made palpation of the apex beat impossible, and the emphysematous chest rendered percussion of the heart unreliable. Sometimes cardiac enlargement was suggested by palpable pulsation immediately below the xiphisternum in the epigastrium. The heart sounds were usually of poor quality, the second sound at the apex often being louder than the first, and in some gallop rhythm further indicated cardiac embarrassment. Cervical venous engorgement could not be determined with any degree of certainty because of the short, stumpy neck and restlessness in most infants. The liver edge was invariably three or four fingerbreadths below the costal margin, but could be overlooked unless palpation was begun well down in the lower half of the abdomen below the liver edge. The spleen was palpable in a few patients. Subcutaneous oedema was present in only two patients and in three the urine contained a trace of albumin but no red blood cells or casts. The overall clinical picture was that of an anoxic infant with severe circulatory failure and considerable respiratory distress. The clinical features of gross liver enlargement and minimal pulmonary congestion suggest that the cardiac failure predominantly affected the right ventricle.

Radiological examination of the chest showed gross uniform cardiac enlargement. In contrast to the large heart the lung fields were usually not congested, but were often hazy; this appearance suggested some oedema. In several patients small bilateral pleural effusions were noted, and one patient had an area of radio-opacity at one lung base suggesting either collapse or consolidation. The electrocardiogram showed serious impairment of myocardial function, the significant abnormal features being low voltage QRS complexes, especially in lead $I$, and low, iso-electric or inverted $T$ waves in all three standard leads.

Necropsy showed that all hearts were dilated and the walls were usually thicker than normal. The only other macroscopic abnormalities were flabbiness of the heart muscle and irregular reddish or pale streaking in the muscle wall. With one exception the pericardium, endocardium and valves were all normal. Histological examination revealed widespread but often patchy damage of the myocardium, and an associated inflammatory reaction with neutrophil and eosinophil polymorphonuclear leucocytes, lymphocytes and macrophages.

Case 1. J.O'B., a boy aged 10 months, was admitted to the Children's Hospital on October 28, 1949, with a history of normal progress until three days previously when he was noted to be pale and rather drowsy. The 
next morning he vomited and periodically throughout the day cried out as if in pain. These symptoms persisted and on the third day his respirations became rapid and grunting. Both parents and a brother aged 2 years were well and there was no contact with any infectious disease. Examination showed a well developed, slightly cyanosed infant who looked very ill and exhausted. The temperature was $98.4^{\circ} \mathrm{F}$., the pulse rate 150 per minute, weak and thready, the blood pressure $7050 \mathrm{~mm}$. $\mathrm{Hg}$ and the respirations 80 per minute and grunting. The extremities were cold and mottled with extremely sluggish capillary circulation. The apex beat could not be felt, the heart did not seem to be enlarged by percussion, the sounds were distant and no murmur was heard. The liver was enlarged three fingerbreadths below the costal margin and there was no subcutaneous oedema or venous engorgement in the neck. The lungs were clinically clear. the urine and blood examination normal. The baby died after $\mathbf{2 4}$ hours without a clinical diagnosis being established.

Necropsy showed a symmetrically enlarged heart, congested and oedematous lungs and some serous fluid in both pleural cavities. The liver was enlarged and pale. The myocardium was diffusely infiltrated with leucocytes, mainly lymphocytes and plasma cells. These cells were aggregated around foci of muscle fragmentation. The liver showed patchy centrilobular necrosis with engorgement of the capillaries.

Case 2. E.D., a girl aged 15 months (weight $18 \frac{1}{2} \mathrm{lb}$.), was admitted to the Children's Hospital on June 19, 1950. Two weeks previously she had been vaguely ill and then for one week she had a slight cough with rapid breathing. Her parents, brothers and sister were well and she had no contact with any infectious disease. The infant was pale, looked ill, and the lips and extremities were slightly cyanosed. The respiratory rate was $90-100$ per minute, the temperature $99^{\circ} F$., the pulse weak and thready at 170 per minute. The apex beat was difficult to define but forcible cardiac pulsation was present under the xiphisternum. No murmur was heard but gallop rhythm was present at the apex. The liver edge was palpable three fingerbreadths below the right costal margin and the tip of the spleen was palpable. The chest was rather emphysematous and a few fine râles were heard at both lung bases. Slight oedema was present in both feet and sacrum. The heart was considerably enlarged radiologically and the lung fields were clear. The urine did not contain albumin. Digitalis folium, grains 4 by mouth over 24 hours, and then grains $\frac{1}{2}$ daily, was given together with oxygen. Forty-eight hours later the infant was very much improved. The colour was good, the pulse rate slower at 120 per minute, and the respiratory rate 36 per minute. The oedema had cleared, and the liver edge was palpable one-and-a-half fingerbreadths below the costal margin. Radiological examination five days after the beginning of treatment showed a heart which was much smaller. Digitalis was discontinued after 17 days. During the next two years the child developed normally and in August, 1952, the heart was normal clinically, radiologically and electrocardiographically.

Case 3. B.S., a girl aged $5 \frac{1}{2}$ years, was admitted on
July 17, 1951, to the Hamilton Base Hospital. She had been off colour for five days with a sore throat and anorexia, had vomited for two days and had several convulsions on the day before admission. Both parents, her sister aged 3 years and brother aged 2 months. were well. The child had faucial diphtheria when aged 2 years. with palatal palsy but no evidence of cardiac involvement; recovery was complete, and she had been well since. Examination showed a healthy, well developed child. The temperature was $99^{\circ} \mathrm{F}$., the pulse regular with good volume at 80 per minute, and the respirations 24 per minute. Slight tonsillitis was present and a throat swab grew Streptococcus viridans. The heart was clinically normal in size, the sounds regular and clear, and no bruits were heard. The blood pressure was $9050 \mathrm{~mm}$. $\mathrm{Hg}$. The lungs were clear and the liver was not enlarged. The urine had a specific gravity of 1020 , contained a trace of albumin, but was microscopically normal. A diagnosis of upper respiratory tract infection with tonsillitis and albuminuria was made, and treatment with penicillin. 100,000 units six-hourly, was given. The child's condition remained unchanged for 36 hours when unexpectedly at night the ward sister heard her cry out and observed a spasm of the arms and arching of the back. This spasm lasted approximately a minute, after which the child was pale, collapsed and sweating. Examination showed a shocked child, whose pulse was impalpable and whose blood pressure could not be recorded. The heart was not enlarged clinically. The ventricular rate was slow and slightly irregular with a rate of 20 to 30 per minute and faint auricular sounds were heard at 130 per minute. No venous engorgement in the neck or subcutaneous oedema was noted. The liver edge was palpable three fingerbreadths below the costal margin. An electrocardiogram showed a complete heart block (Fig. 1A). Treatment with oxygen, ephedrine, and adrenalin was ineffective, and gradually the circulation became worse. cyanosis pronounced, and the child died eight hours later.

Necropsy showed a slightly dilated heart with a flabby myocardium. The lungs were engorged and the liver enlarged with a nutmeg appearance. Histologically the myocardium showed patchy fragmentation of the muscle fibres accompanied by a cellular reaction consisting of eosinophil and neutrophil polymorphonuclear leucocytes, macrophages and lymphocytes (Fig. 1B).

Case 4. S.H., a girl aged 14 months (weight $16 \frac{1}{2} \mathrm{lb}$.). was admitted to the Children's Hospital on August 20. 1951, with a 'cold', slight fever and nasal discharge for five days. For two days her breathing had been wheezy. grunting and rapid, and her colour cyanotic. There was no contact with any infectious disease. Her past history revealed that at the age of 6 weeks she developed a staphylococcal lung infection which persisted in a subacute form over six months despite adequate treatment with penicillin and later with aureomycin. During this time she failed to thrive, her stools became greasy and a clinical diagnosis of fibrocystic disease of the pancreas was made. Skiagrams of the heart and lungs from the age of 6 weeks to 10 months showed a heart of normal size and lung changes compatible with bronchitis and 


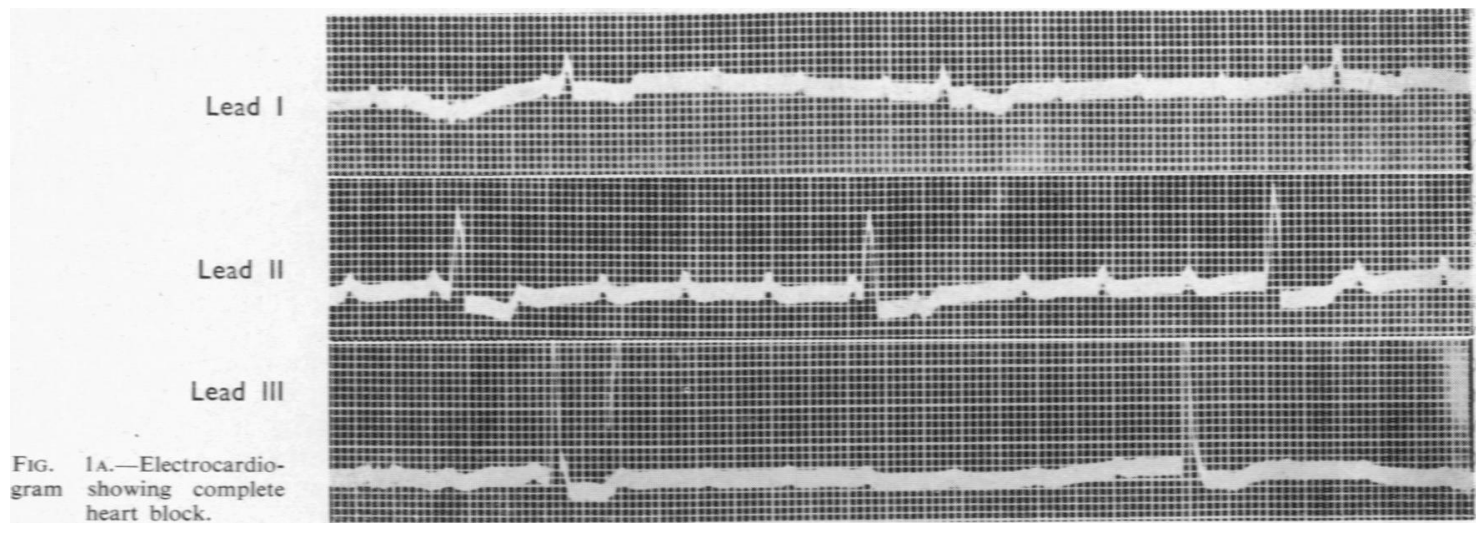

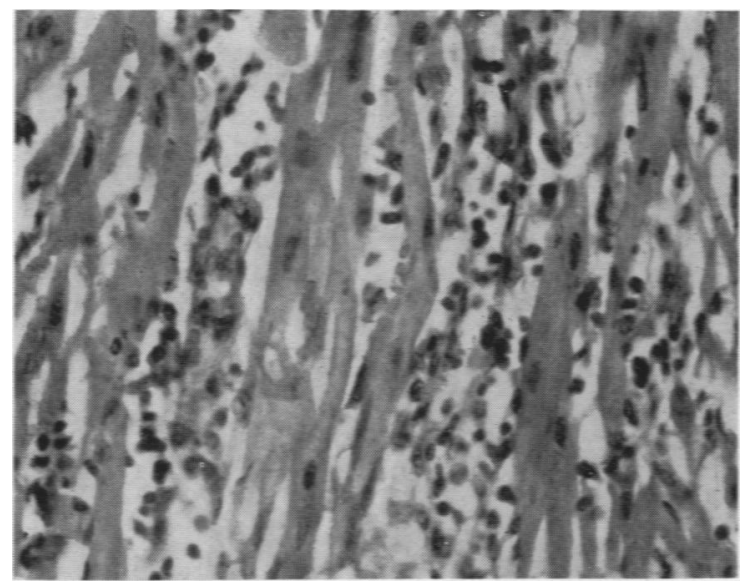

Fig. 1B.-Section from the interventricular septum showing diffuse muscle cell damage and cellular infiltration. $\cdot 110$.

patchy pneumonitis. At 10 months of age she weighed only $13 \mathrm{lb}$. $10 \mathrm{oz}$., but over the next four months she improved steadily, took her feeds well, gained weight and her cough cleared. Examination on admission revealed a distressed, sick infant with rapid, wheezy respirations at 56 per minute, a temperature of $99^{\circ} \mathrm{F}$., and pulse rate of 136 per minute. Clinically the heart was considered to be normal, but a skiagram of the chest showed gross symmetrical enlargement of the heart with slight pulmonary congestion. Scattered râles were heard at both lung bases with expiratory ronchi, and abdominal examination revealed no abnormality. A diagnosis of bronchopneumonia was made and treatment with penicillin and streptomycin given. The infant unexpectedly collapsed and died two days later.

Necropsy revealed a dilated heart with flabby myocardium. The lungs showed macroscopic and histological changes of bronchiolitis with pulmonary congestion at the bases. The pancreas was normal. Microscopically the heart muscle showed much fragmentation and diffuse mononuclear cell infiltration.
Case 5. J.L., a girl, aged $4 \frac{1}{2}$ years, was admitted to the Hamilton Base Hospital on September 10, 1951, with a provisional diagnosis of acute appendicitis. The child was well until the day of admission when she developed abdominal pain, lost her appetite and vomited. Examination disclosed a sick girl with a slightly enlarged heart, a soft, apical, systolic bruit and an enlarged, tender liver. The following day the liver was further enlarged to three fingerbreadths below the costal margin and the apex beat was in the fifth left intercostal space outside the nipple line. The temperature was $102=\mathrm{F}$., the pulse rate 140 per minute, the respirations rapid and grunting at $\mathbf{5 0}$ per minute, and the urine normal. Over the next three days the child's condition slowly deteriorated. The respirations were then rapid and grunting. the liver enlarged four fingerbreadths below the costal margin and crepitations appeared at the left lung base. Digoxin, $0.25 \mathrm{mg}$. statim and 0.125 mg. six-hourly, was begun, but death occurred 20 hours later.

Necropsy revealed a dilated heart (weight, 135 g.) with pale but firm muscle and a small serous exudate with fibrin clot in the pericardial sac. The lungs were congested and oedematous and the enlarged liver (weight, 765 g.) had a nutmeg appearance. Histologically widespread foci of myofibril damage with an accompanying cellular reaction of lymphocytes, macrophages and scattered polymorphonuclear leucocytes were found throughout the myocardium. In the region of the attachment of the mitral valve to the atrioventricular ring there was a relatively large area of infiltration with polymorphonuclear leucocytes and a small area of lymphocytic infiltration was present near the base of the valve.

Case 6. J.B., a boy aged 3 weeks (weight 6 lb.), was admitted to the Children's Hospital on February 9, 1952. He was a normal full term baby, the first child of healthy parents, and the pregnancy was normal. He was well until three days before admission to hospital when he was noted to be drowsy and pale, and he vomited several times. Twenty-four hours before admission his breathing became rapid, and grunting and cyanosis developed. During the day he passed five loose stcols. Examination showed a pale, slightly cyanosed baby with rapid. regular, 

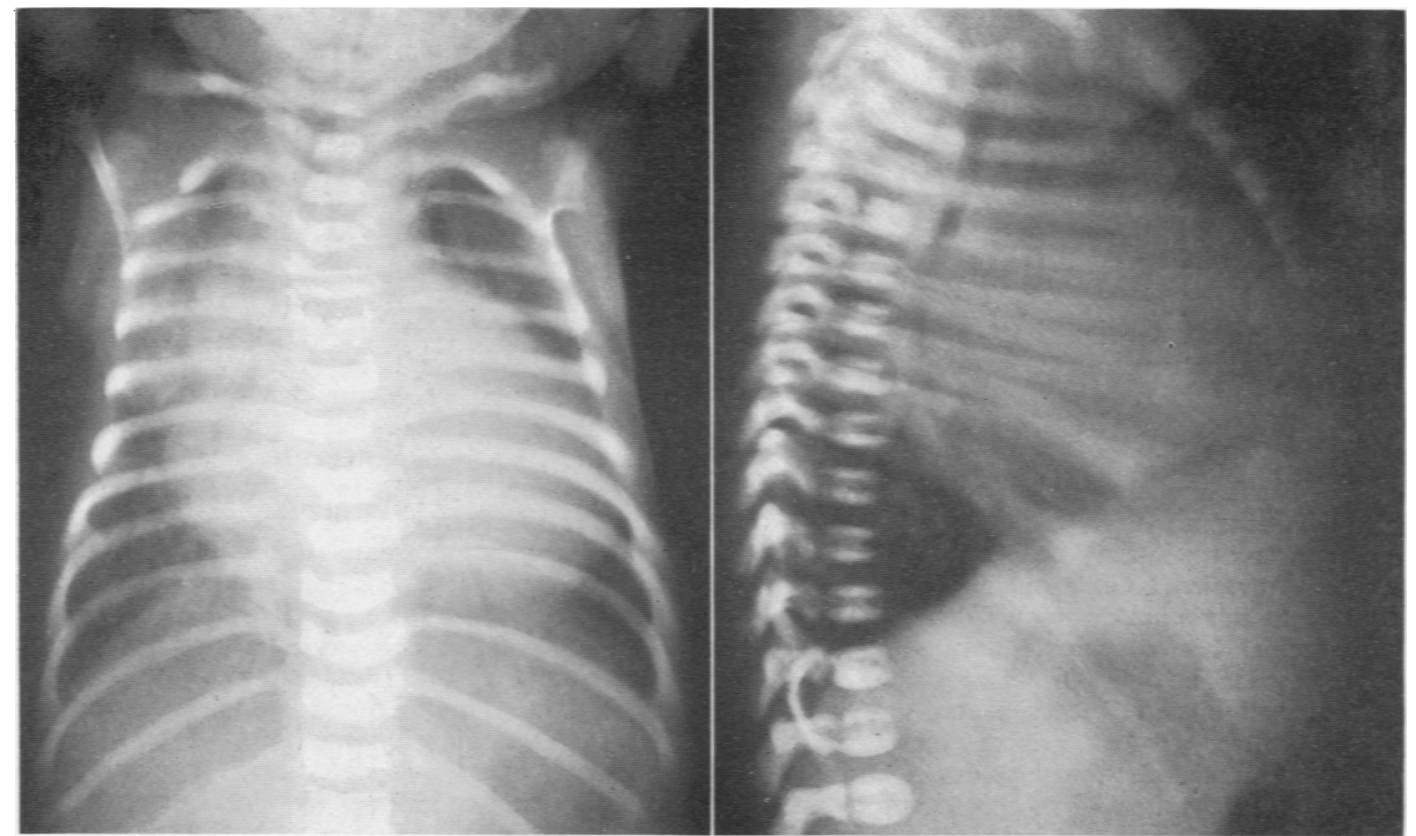

FIG. 2A.-Radiograph of chest showing gross symmetrical cardiac enlargement with slight pulmonary engorgement. Note the low flat diaphragm. the anterior displacement of the sternum and the posterior displacement of the trachea.

slightly grunting, shallow respirations at 60 per minute. The extremities were cold and mottled, the temperature $97^{\circ} \mathrm{F}$., and the pulse was weak and thready at 160 per minute. The cardiac size could not be assessed clinically. Heart sounds were regular and faint, and no murmur but gallop rhythm was heard. The chest was barrel-shaped and scattered crepitations were heard over both lung fields. The liver was three fingerbreadths below the costal margin and the tip of the spleen was felt. There was no peripheral oedema, or venous engorgement in the neck. The urine was clear of albumin, cells and casts. A radiograph of the chest showed a grossly enlarged heart, which largely obscured slightly congested lung fields and displaced the trachea and oesophagus posteriorly (Fig. 2A). Digoxin, 0.05 mg. intramuscularly, and oxygen were given, but death occurred four hours later.

Necropsy revealed a symmetrically enlarged, dilated heart, weighing $48 \mathrm{~g}$. The muscle was flabby and appeared mottled with dark and pale areas. The lungs were engorged with some consolidation at the right base and the liver was enlarged and congested (weight, $116 \mathrm{~g}$.). Microscopic examination of the heart showed that the myofibrils had disappeared from extensive areas leaving a reticular structure. Some cellular reaction with lymphocytes, plasma cells and fibroblasts was noted at the periphery of these lesions (Fig. 2B). It appeared that these lesions had been present for a longer time than was indicated by the infant's symptoms.

Case 7. S.A., a boy aged 3 weeks, was admitted to the Children 's Hospital on March 4, 1952. He was a normal full term baby of healthy parents and the pregnancy had been normal. He had made good progress until 11 days

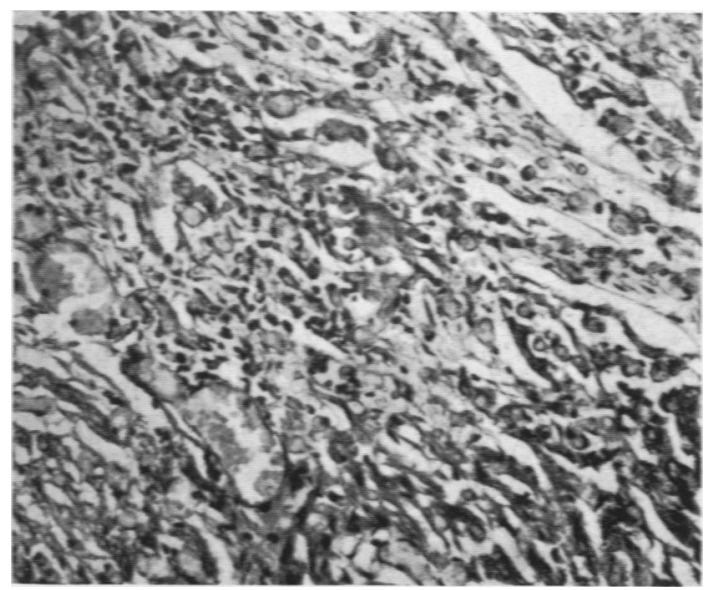

FK. 2B-Histological section of the left ventricular wall showing gross muscle cell necrosis and vacuolation with cellular infiltration. $\times 110$.

old when he became off colour and later his breathing was rapid and grunting and his colour greyish. Periodically he screamed and drew up his legs as if in pain. Two days later he temporarily improved but was not well. For the next five days his condition was unchanged but then he developed a cough, and became pale and listless. Later he was cyanosed with rapid, grunting breathing. Examination showed an ill, cyanosed baby with a respiratory rate of 70 per minute, a temperature of $95^{\circ} \mathrm{F}$., 
a pulse rate of 160 per minute, cold mottled extremities and poor capillary circulation. The heart was clinically enlarged with pulsation in the fifth left intercostal space outside the nipple line and in the epigastrium under the xiphisternum. The heart sounds were faint but no murmurs were heard. No cervical venous congestion was observed. The chest was slightly emphysematous and scattered crepitations were heard more on the right side than the left. The liver was palpable three fingerbreadths below the costal margin and the tip of the spleen was palpable. No subcutaneous oedema was noted. A radiograph of the chest showed gross uniform cardiac enlargement with mild congestive changes in the lung fields, and a radio-opacity in the right lower lung. A clinical diagnosis of congenital heart disease with congestive cardiac failure and respiratory tract infection was made and treatment with oxygen, penicillin and streptomycin given, but the infant died eight hours later.

Necropsy revealed an enlarged heart (weight, $47 \mathrm{~g}$.) with the right side more dilated than the left, and some small petechial haemorrhages along the line of the coronary vessels. The lungs were congested and oedematous and small bilateral serous pleural effusions were present. The liver was enlarged and congested with a nutmeg appearance. Histological examination of the heart showed patchy areas of myofibril damage throughout the left ventricle and to a lesser extent in the other chambers. The cellular reaction consisted mainly of lymphocytes and polymorphonuclear leucocytes.

Case 8. R.D., a girl aged 15 months, was admitted to the Children 's Hospital on March 7, 1952, with a history of malaise and slight fever for one week, and rapid grunting breathing, pallor and cyanosis for four days. The baby had been treated for pneumonia for three days. Examination showed a very ill, restless, pale, slightly cyanosed, apprehensive-looking baby. The respiratory rate was 50 per minute, the chest was barrel-shaped and no crepitations were heard. The extremities were cold and mottled, the capillary circulation very poor, the pulse rate 150 per minute, and the blood pressure $7050 \mathrm{~mm}$. Hg. Cardiac pulsation was felt in the fifth left intercostal space outside the nipple line, in the third and fourth right intercostal spaces close to the sternum and in the epigastrium. Gallop rhythm and distant heart sounds were heard but no murmur was present. The liver edge was felt four fingerbreadths below the costal margin and the spleen was just palpable. No subcutaneous oedema was detected. There was moderate albuminuria but no cells or casts in the urine. A radiograph of the chest showed gross symmetrical cardiac enlargement, slightly hazy lung fields which were not congested, and small bilateral pleural effusions. The electrocardiogram showed a rate of 150 per minute, low voltage ventricular complexes and isoelectric $T$ waves in all three standard leads. Digoxin, $0.2 \mathrm{mg}$. statim was given intramuscularly, and $0.2 \mathrm{mg}$. in six hours together with oxygen. The infant did not improve and died suddenly eight hours after admission.

Necropsy revealed a flabby, enlarged heart (weight, 95 g.) with some oedema of the lungs and moderate enlargement and congestion of the liver. Histologically the heart showed diffusely scattered foci of fragmented and oedematous muscle fibres. Lymphocytes and macrophages infiltrated the entire muscle but were aggregated particularly in and around the foci of muscle damage. The lungs showed diffuse areas of haemorrhage with mononuclear cells and macrophages in the alveoli. The liver was congested with some fat accumulation.

Case 9. J.B., a girl aged 12 days, weighing $5 \mathrm{lb} .12 \mathrm{oz}$., was admitted to the Children's Hospital on October 8, 1952. The past history was that of a normal pregnancy and delivery, and the baby had sucked well and madeco satisfactory progress for the first eight days in the? maternity hospital. She went home on the ninth day when she refused her feeds and her breathing was noted to be noisy. The following day the skin was cold and slightly mottled. Over the next two days these symptoms persisted and her stools were noted to be greenish-black and loose. On the day of admission the feet and hands

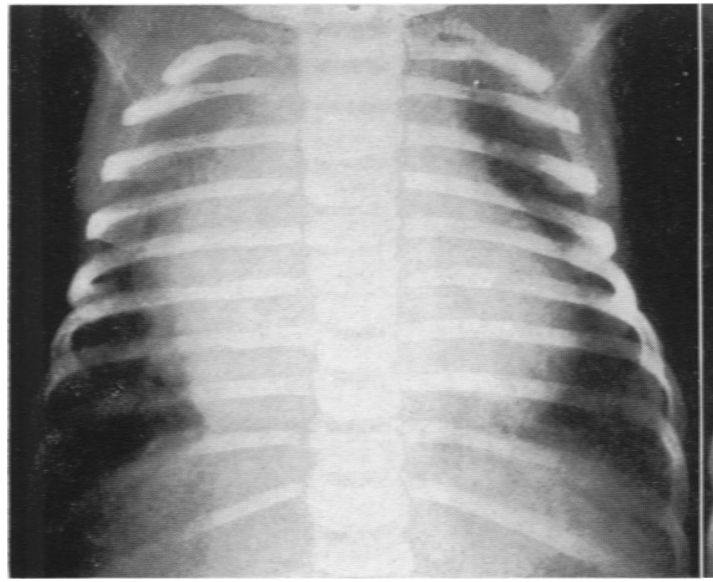

Fig. 3A.-Radiograph of the chest on admission on October 8 showing gross symmetrical cardiac endargement and slight pulmonary oedema and congestion, and small bilateral pleural effusions.

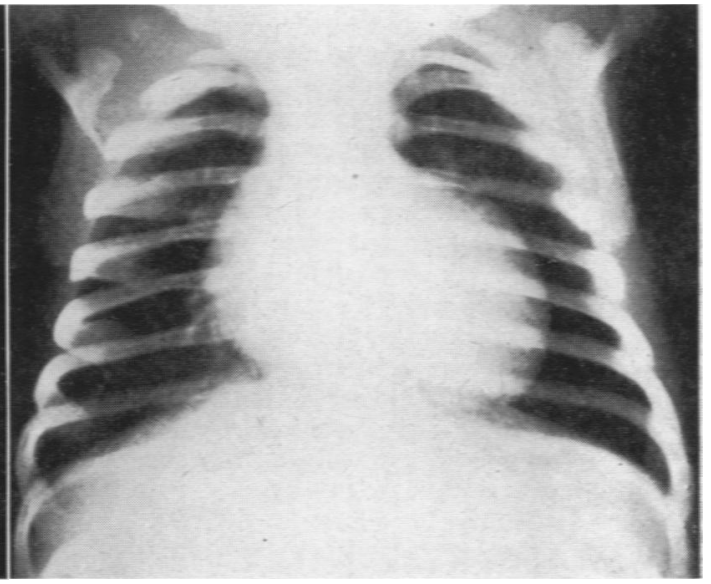

FiG. 3B.-Radiograph on October 24, 16 days later. Normal. 


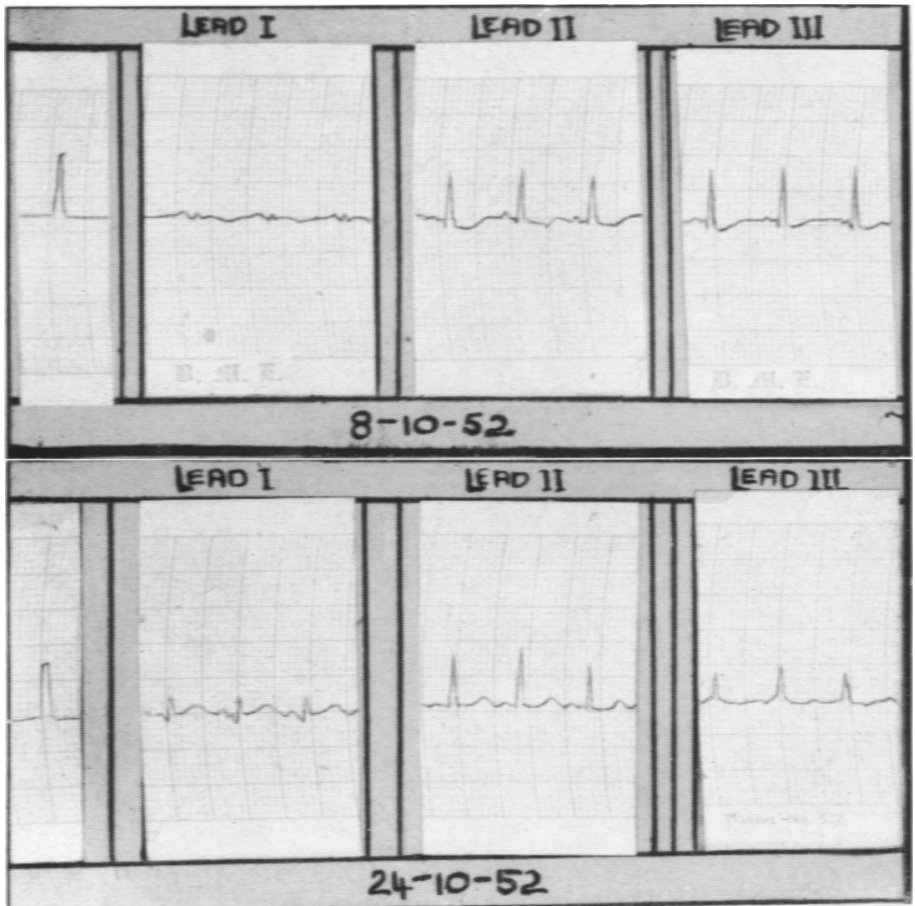

FKG. 3c.-Electrocardiogram on admission on October 8 showing low amplitude QRS complex in lead I and inverted $T$ waves in leads I, II and III.

FIG. 3D.-Electrocardiogram on October 24, 16 days later, upright $T$ waves in leads I and II, increased amplitude of QRS complex in lead $\mathrm{I}$.

became swollen. There was no known contact with infection either in the hospital or at home. Examination showed a small, pale, mottled baby with generalized oedema. The respirations were rapid at 70 to 80 per minute and grunting with some retraction of the lower rib margins. The temperature was $96^{\circ} \mathrm{F}$., the pulse rate 150-160 per minute, the extremities cold and the capillary circulation poor. The heart size was difficult to determine clinically but forcible cardiac pulsation was felt in the epigastrium below the xiphisternum. A gallop rhythm but no murmur was heard. The breath sounds were loud and harsh but no crepitations were heard. The liver edge was palpable two finger-breadths below the right costal margin. The urine showed a trace of albumin but microscopically was clear. The cerebrospinal fluid was normal. A radiograph of the chest showed gross symmetrical cardiac enlargement with slight increase in the bronchovascular pattern in the lungs. The electrocardiogram revealed tachycardia of 150 per minute, normal $P$ waves and PR interval, low QRS complexes in all leads, but especially in lead $I$, and inverted $T$ waves in all leads (Fig. 3A and C).

Digitalis folium, grain $\frac{1}{2}$ statim and grain $\frac{1}{4}$ six-hourly for four doses and then grain $\frac{1}{4}$ daily, was administered together with oxygen. After 24 hours' treatment she was much improved. The colour was normal, the respirations easier but still rapid, while the oedema and gallop rhythm were unchanged. The urine was now clear of albumin. In 48 hours the infant had a good cry and sucked well. The liver was now palpable only one fingerbreadth below the right costal margin and the gallop rhythm and oedema had disappeared. Rapid improvement continued and by the sixth day the heart was much smaller radiographically, the pulse rate 130 per minute and respiratory rate 36 per minute. An electrocardiogram showed increased amplitude of the QRS complex with small upright $T$ waves in lead I but there was no other significant change in leads II and III. Improvement was maintained on digitalis and 16 days after treatment began the baby was clinically normal. The heart was normal in size radiologically and the electrocardiogram showed normal $T$ waves in leads I and II but the QRS complex in lead I was still low (Figs. 3B and D). After six weeks treatment the baby weighed $8 \frac{1}{2} \mathrm{lb}$., was clinically nor$\mathrm{mal}$ : the radiograph of the heart was normal and the electrocardiogram showed upright $\mathrm{T}$ waves in leads I and II and further increase in the amplitude of the QRS complex in lead I.

\section{Subacute and Chronic Myocarditis}

In contrast to the group of nine patients with an acute illness and rapid onset of severe heart failure, five patients had an illness of more gradual and insidious onset before cardiac failure developed. Four of these five patients recovered but all have residual myocardial damage. The prodromal symptoms were those of general malaise or vague illhealth for a month or more before symptoms directly referable to myocardial failure developed. The outstanding and constant symptoms of failure were vomiting, rapid, grunting breathing, pallor, cyanosis and later oedema. The physical signs present in all were cardiac enlargement, tachycardia, gallop rhythm, gross liver enlargement and, by contrast, minimal signs of pulmonary congestion. Radiologically there was gross but symmetrical cardiac enlargement, hazy and slightly congested lung fields and often small bilateral pleural effusions. The electrocardiographic findings were the same as in the acute type. The $P$ waves and PR interval were normal, the amplitude of the QRS complex was low in all leads but especially in lead $I$, and the $T$ waves were either flat, isoelectric or inverted. The course of the disease was prolonged. All responded to digitalis therapy, but relapsed at varying intervals 
following its cessation. All have permanent cardiac damage and in two there is evidence of a mitral valvular lesion. The pathological lesions in the patient who died were essentially the same as those in the acute group. While it is not possible to prove that the pathological lesion in the four survivors was the same, the clinical, radiological and electrocardiographic features were identical.

Case 10. M.H., a girl aged 3 years. weight $34 \mathrm{lb}$., was admitted to the Children's Hospital on August 4. 1949. One month previously she had contracted an upper respiratory tract infection and had not been well since. Her brother and both parents had also contracted a similar infection. One week before admission she developed abdominal pain. flatulence and vomiting. Distressed breathing. pallor and pain in the chest for one day were the cause of her admission to hospital, She was a pale, cyanosed child with rapid respirations of 50 per minute, and a weak, rapid pulse of 150 per minute. Her temperature was $98^{\circ} \mathrm{F}$., and her blood pressure $7550 \mathrm{~mm}$. Hg. The veins in the neck were engorged and the heart clinically enlarged. The heart sounds were soft, and gallop rhythm but no bruit was heard. The chest was clear clinically, the liver enlarged four fingerbreadths below the costal margin and the sacrum and feet pitted on pressure.

Radiologically the heart was very large and by contrast the lung fields were only slightly congested and hazy. The electrocardiogram showed low QRS complexes and flat $T$ waves in all leads. No pathogenic organisms were cultured from the throat swab and the antistreptolysin titre in the serum was less than 25 units per $\mathrm{ml}$. The urine was normal chemically and microscopically. Treatment with oxygen and digoxin, $1 \mathrm{mg}$. during the first day, $0.4 \mathrm{mg}$. during the second day, and then $\frac{1}{2}$ grain digitalis folium daily, produced rapid relief of her symptoms. After one week she was well, the heart rate was 80 per minute, but the heart was still somewhat enlarged. After four weeks on digitalis she was sent home and was well for five weeks when she caught a "cold', after which she again became off colour, complained of abdominal pain and was short of breath. All the signs of cardiac failure recurred and she was re-admitted to hospital. Again culture of the throat swab yielded no pathogenic organisms and the serum antistreptolysin titre was less than 50 units per $\mathrm{ml}$. Treatment with digitalis again produced a good response, and after one month's treatment she was sent home clinically well but with an enlarged heart. Seven months after the original attack a slightly rough, 'blowing' apical systolic murmur appeared for the first time and this has persisted unchanged to August, 1952. She has been well during this time, enjoys full activity, but her heart is still slightly enlarged both clinically and radiologically. Her electrocardiogram is normal.

Case 11. F.P., a girl aged 20 months (weight $22 \frac{1}{2} \mathrm{lb}$.), was admitted to the Children's Hospital on August 29, 1950 , with a history of being off colour for one month. One week before admission she began to vomit and passed very little urine. Her face and feet gradually became swollen and her breathing rapid and grunting. She was the only child of healthy parents, had always been well and had not been in contact with any person with an infectious disease. Examination showed an ill. cyanosed infant with rapid, grunting respirations at 60 per minute, a weak, thready pulse of 160 per minute, cold, mottled extremities and a temperature of $99^{\circ} \mathrm{F}$. The heart was enlarged slinically. and gallop rhythm but no murmur was heard. The blood pressure was 9070 . The liver edge was felt three and one-half fingerbreadths below the costal margin, crepitations were heard at both lung bases and slight oedema of legs. sacrum, hands and face was noted. The urine contained a trace of albumin. a few red blood cells and an occasional cast for one day. after which it was normal. A radiograph of the chest showed gross symmetrical cardiac enlargement. a small bilateral pleural effusion and a radio-opacity in the right lower zone which was considered to be an area of consolidation (Fig. 4A). The serum antistreptolysin titre was less than 50 units per $\mathrm{ml}$. The electrocardiogram showed a sinus tachycardia of 160 per minute. normal $P$ waves and $P R$ interval. low $Q R S$ complexes and isoelectric $T$ waves in all three standard leads (Fig. 4C). Treatment with digitalis folium, grains 4 for 24 hours, grains 2 daily for two days, and then grain $\frac{1}{2}$ daily, together with oxygen, produced a satisfactory response. In 72 hours she was beginning to play. The pulse rate was now 88 per minute, the respirations 30 per minute and the blood pressure $11086 \mathrm{~mm}$. $\mathrm{Hg}$. The oedema of the face and crepitations in the lungs had cleared, the liver edge was just palpable and she had lost $2 \mathrm{lb}$. in weight.

Digitalis therapy was discontinued after one month, when the infant was well but the heart still enlarged. Two weeks later following an upper respiratory tract infection all the signs and symptoms of congestive failure recurred and the infant was as ill as previously. On this occasion digitalis therapy did not produce a rapid clinical improvement. After ten days failure was still pronounced and 'mersalyl' injections $(0 \cdot 25 \mathrm{ml}$. intramuscularly daily) were begun. While this treatment reduced some of the oedema, the heart still remained large, the rate rapid at 120 per minute, and gallop rhythm was intermittently heard. The liver edge remained three fingerbreadths below the costal margin. During the next eight months, despite digitalis folium, grains $\$$ daily, and 'mersalyl' $0.5 \mathrm{ml}$. on alternate days, the infant remained in a state of chronic cardiac failure. Approximately one year after the illness she began to improve slowly, looked better, the heart rate became slower, the heart size smaller and the liver gradually returned to a normal size. A blowing systolic murmur now developed at the apex. In November, 1952, two years and three months after the initial illness, she was very well. The heart was clinically and radiologically still enlarged, and the electrocardiogram showed low $T$ waves in all leads (see Figs. 4B and D).

Case 12. D.B., a girl aged 6 years, was admitted to the Children's Hospital on August 22, 1950, with the history that she had always been well until five weeks previously when she became listless, lost her appetite, developed a slight dry cough and became breathless on 


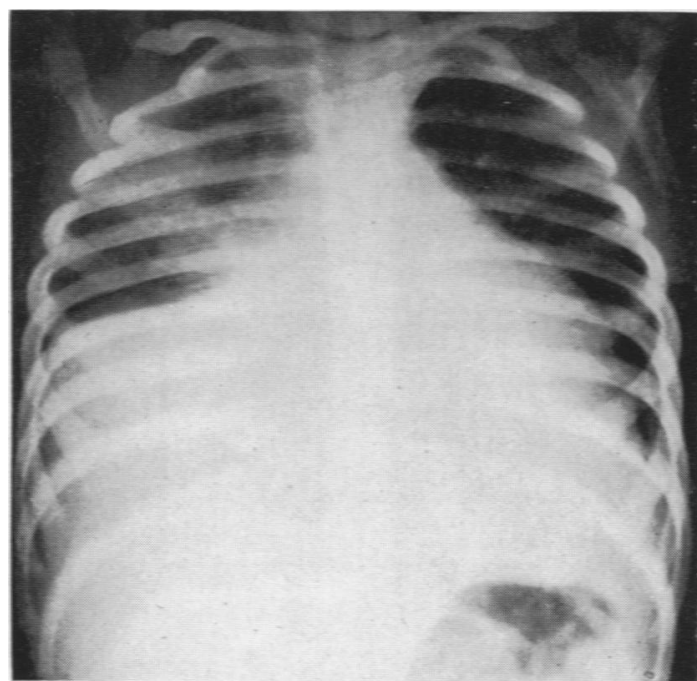

FrG. 4A.-Radiograph of chest on admission on August 20, 1950, showing gross cardiac enlargement, an opacity in the right lower lung field and a small left pleural effusion. The opacity was considered to be the result of a right lower lobe consolidation.

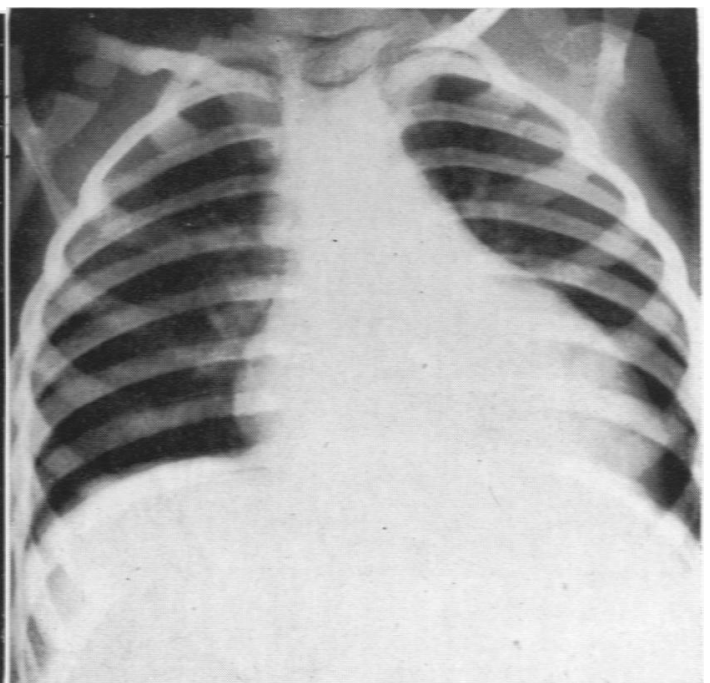

FG. 4B.-Radiograph of June 17, 1952, showing slight cardiac enlargement. Child clinically well but with evidence of a mitral valve lesion.

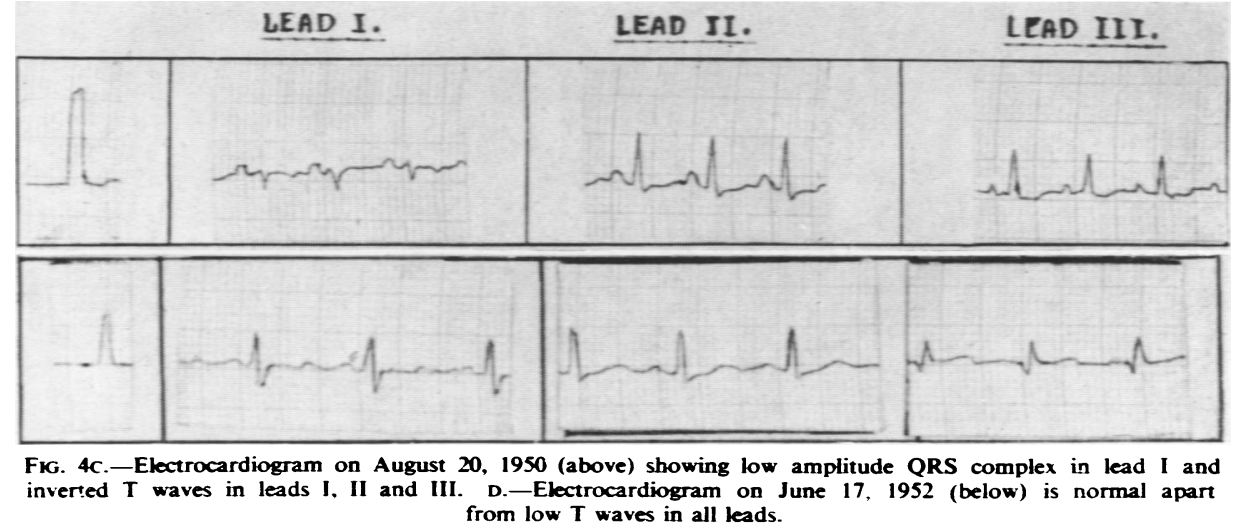

walking. The parents and three other children were well and the patient had not been in contact with any infectious disease. Examination showed a sick child, with a temperature of $98^{-}$F., quiet respirations of 24 per minute, pulse 130 per minute, and a blood pressure of $7550 \mathrm{~mm}$. Hg. The heart was considerably enlarged clinically and gallop rhythm, but no murmur, was heard. The veins in the neck were engorged, the liver enlarged three fingerbreadths below the costal margin, the lungs were clear but the legs and sacrum pitted on pressure. The urine was clear of albumin and cells. A radiograph of the chest showed a grossly enlarged heart but no pulmonary engorgement. The electrocardiogram showed a tachycardia of 120 per minute, normal $P$ waves, normal PR interval, low voltage QRS complexes and very low $T$ waves in all three standard leads. The serum anti- streptolysin titre was below 25 units per $\mathrm{ml}$. and culture of the throat swab did not grow any pathogenic organisms. With digitalis folium, grains 8 over 48 hours and then grain 1.daily, the child rapidly improved so that in three days the heart rate was $\mathbf{8 0}$ per minute, the gallop rhythm and subcutaneous oedema had disappeared and the liver edge was palpable only one fingerbreadth below the costal margin. The electrocardiogram showed that the QRS complexes and $T$ waves were returning to normal. During the next six months she remained well while resting in bed, but the heart remained grossly enlarged. At this time she was allowed up but would not attempt to run or hurry as she became very breathless. The heart remained very enlarged and the pulse rate 90 to 100 per minute. After a period of about six months, i.e. approximately one year after she first became 


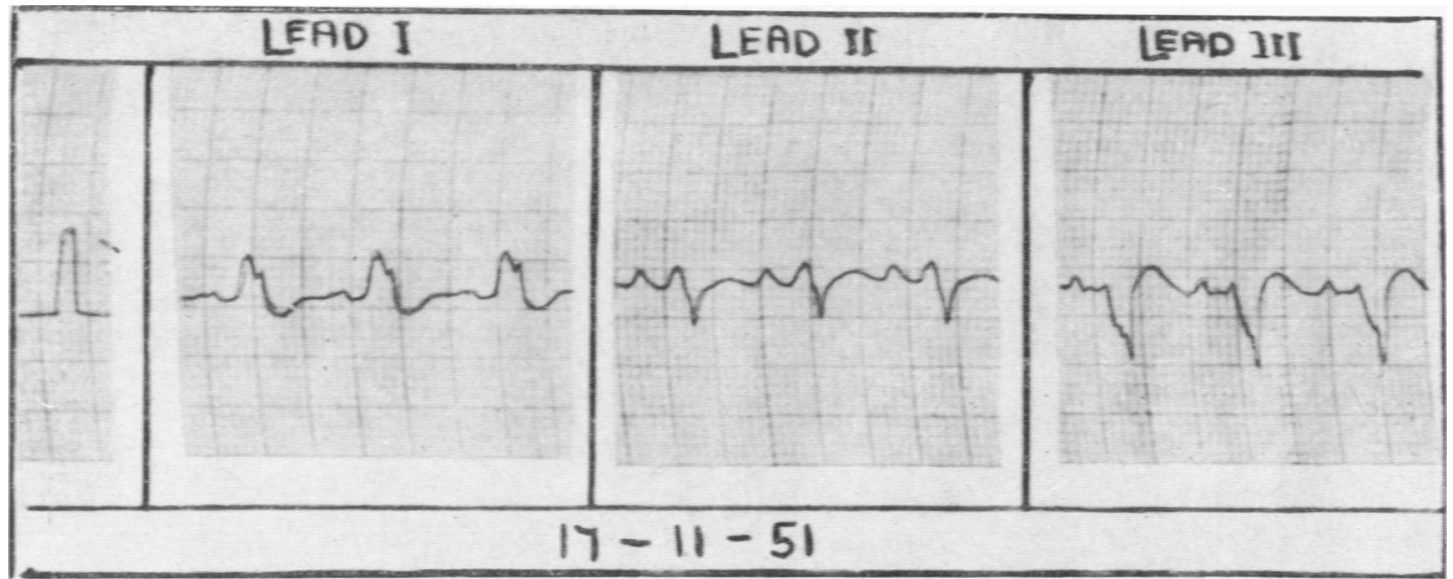

FIG. 5A.-Electrocardiogram showing bundle branch block.

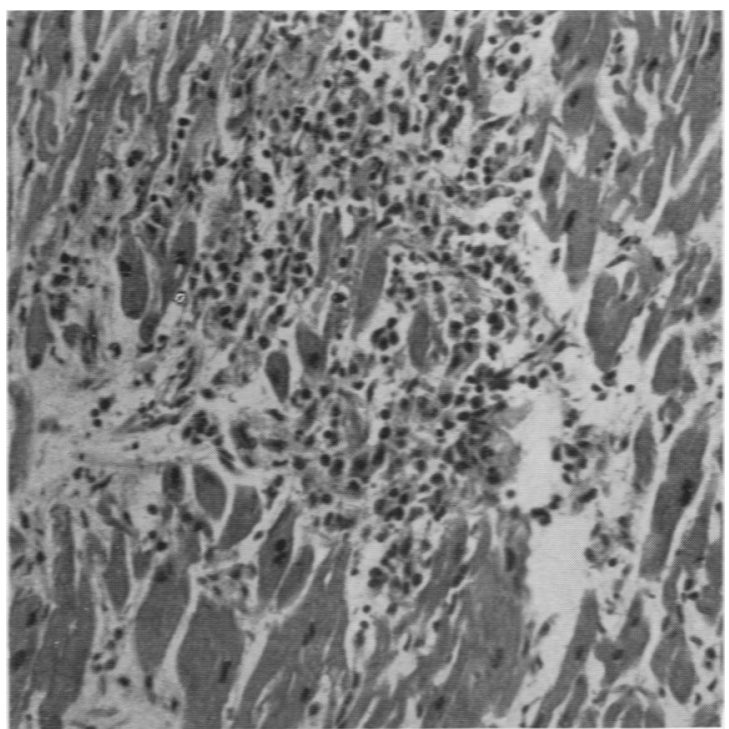

Fig. 5B.-Histological section of ventricular wall showing a patch of muscle cell damage with inflammatory cell reaction. ; 110.

ill, congestive cardiac failure again developed. Even with complete bed rest, digitalis and later 'mersalyl' injections a satisfactory response did not occur. The heart rate remained rapid at 120 per minute, the heart grossly enlarged, the legs and sacrum oedematous and the liver grossly enlarged. An electrocardiogram showed an early bundle branch block (Fig. 5A). The congestion gradually became more marked, the subcutaneous oedema increased, a complete bundle branch block developed and the child died 16 months after she first took ill.

Necropsy revealed gross enlargement of the heart due mainly to dilatation of all chambers. The ventricular walls were a little hypertrophied, the right side more than the left. Histologically the muscle cells showed patchy gross vacuolation while scattered areas of round cell infiltration were noted between the muscle fibres (Fig. 5в). The coronary vessels showed slight thickening of the intima. No Aschoff nodules, endocarditis or pericarditis were observed. The lungs were very oedematous with chronic congestive changes and the liver enlarged with a typical nutmeg appearance.

Case 13. R.O., a girl aged 18 months, was admitted to the Children's Hospital on October 13, 1951, with the history that at the age of 5 months she became off colour, developed a nasal discharge and later on a cough and wheeze. As these symptoms persisted it was considered that she might have had a chronic lung infection. Radiological examination of the chest showed that the only abnormal feature was moderate symmetrical cardiac enlargement. From the age of 7 to 18 months she was well, grew and developed normally but serial clinical and radiological examinations showed that the heart was persistently enlarged. At 18 months of age she insidiously became ill and was admitted shortly after with congestive heart failure. She was moderately dyspnoeic with a respiratory rate of 46 per minute, a pulse rate of 150 per minute and temperature of $99^{\circ} \mathrm{F}$. The heart was grossly enlarged, and gallop rhythm but no bruit was heard. The veins in the neck were engorged, the liver palpable three and one-half fingerbreadths below the costal margin, scattered rhonchi were heard in the chest and the feet and legs were oedematous. A radiograph of the chest revealed gross symmetrical cardiac enlargement and only slight engorgement and haziness of the lung fields. An electrocardiogram showed a heart rate of 160 , normal $P$ waves and PR interval, normal $Q R S$ complexes but isoelectric $\mathrm{T}$ waves. Treatment with digitalis folium, $7 \frac{1}{2}$ grains for three days with 'mersalyl', $0.5 \mathrm{ml}$. daily, resulted in rapid improvement. The heart rate fell to 80 per minute, the gallop rhythm disappeared, the liver receded to two fingerbreadths and the oedema disappeared. During the next week the child continued to improve. However, the heart remained enlarged, and the liver edge was palpable one fingerbreadth below the 
costal margin. Digitalis was discontinued after three weeks but convalescence was interrupted by an intercurrent respiratory tract infection. She was sent home six weeks after admission.

During the next eight months she was not well. Periodic clinical examinations from November, 1951, to July, 1952, showed a moderately enlarged heart, with at times a triple rhythm, and the liver edge was palpable two fingerbreadths below the costal margin. Gradually the congestive failure became worse and she was again admitted to hospital on July 30 , 1952, with a grossly enlarged heart and liver. The electrocardiogram showed a tachycardia with isoelectric $T$ waves. Treatment with digitalis produced a very satisfactory response and by November, 1952, the only abnormal findings were a moderately enlarged heart and flat $T$ waves in the electrocardiogram.

Case 14. T.W., a boy aged 17 months, was admitted to the Children's Hospital on August 12, 1952, having progressed normally until nine weeks previously when he became off colour and feverish. No other members of the household were sick. Later he vomited and his breathing became rapid and grunting. His family doctor then made a diagnosis of bronchopneumonia and prescribed sulphonamide tablets. Over the next two weeks he seemed to slowly improve but one week later the vomiting and distressed breathing recurred. Again a diagnosis of bronchopneumonia was made and treatment with penicillin given without improvement. The colour remained poor, the respirations rapid, and one month later the feet, hands and face gradually became swollen and he passed only small quantities of urine. Examination showed a flushed, sick-looking infant with a temperature of $100^{\circ} \mathrm{F}$., pulse rate 130 per minute and respirations of 50 per minute, with some retraction of the lower part of the thorax on inspiration. The heart was enlarged clinically, the sounds were distant and gallop rhythm was heard. The veins in the neck were engorged, and the chest was emphysematous with a few crepitations over the right side anteriorly. The liver was enlarged four fingerbreadths below the right costal margin. Generalized oedema, including swelling of the face, was present. A radiograph of the chest showed gross symmetrical enlargement of the heart, with slight congestion and haziness of the lung fields and small bilateral pleural effusions. An electrocardiogram showed normal $\mathbf{P}$ waves, and $\mathbf{P R}$ interval, almost isoelectric $T$ waves and low voltage QRS complexes. The urine was clear chemically and microscopically. Digitalis folium, grains $6 \frac{1}{2}$, was given for 24 hours and then grain $\frac{1}{2}$ daily. Within 48 hours the infant was very much better. The pulse rate was 80 per minute and the respirations were quiet at 20 per minute. The oedema had decreased, the weight had fallen by $2 \frac{1}{2} \mathrm{lb}$., and the liver was palpable only one-half fingerbreadths below the costal margin. Rapid and satisfactory progress was maintained so that digitalis was discontinued after 17 days. The electrocardiogram was now normal, the heart very much smaller radiographically, the liver edge just palpable, and all oedema had disappeared. As the child continued to remain well he was allowed to go home six weeks after treatment began. the only abnormal finding being slight cardiac enlargement.

On arrival home he seemed to contract a slight 'cold' which cleared in three days. One week later he became irritable and vomited and then began breathing rapidly and three days later, on September 1, 1952, was readmitted with congestive cardiac failure. The heart was considerably enlarged, gallop rhythm was audible and the liver palpable three fingerbreadths below the right costal margin. The electrocardiogram showed a heart rate of 160 per minute, with low ventricular complexes especially in lead $I$ and isoelectric $T$ waves in all leads. Response to digitalis therapy was prompt and he steadily improved. Eleven weeks later while still on digitalis he was very well, but the heart was still moderately enlarged and the electrocardiogram still showed rather low QRS complexes but normal $T$ waves.

\section{Discussion}

Aetiology. The aetiology of the myocarditis in this group of children is unknown. The role that infection plays is uncertain and on the available evidence impossible to decide. Clinically three of the 14 patients had a mild associated respiratory tract infection and one other of those who died had post-mortem evidence of pneumonia. While detailed bacteriological and virus investigation was not conducted either during life or after death, 10 of the 14 patients had neither clinical nor pathological evidence of infection, and interrogation failed to determine a contact source of infection.

Identical myocardial lesions have been found associated with many different infections and Saphir with his co-workers give a full review in a series of papers (Saphir, 1941, 1942; Saphir and Wile, 1942: Saphir, Wile and Reingold, 1944; Gore and Saphir, 1947). In infancy and childhood the infections most commonly associated with myocarditis are diphtheria, poliomyelitis and measles, but myocarditis has been found occasionally with almost every infection. The similarity of the pathological lesions in our patients to those described with a known infection suggests, but does not establish, an infective aetiological basis.

It might be considered that the four patients with subacute myocarditis who recovered, and especially the two who developed an apical systolic bruit, did not have idiopathic but rheumatic myocarditis. Such a diagnosis is highly improbable on the grounds of the very young age of the patients, the complete lack of other clinical manifestations of rheumatic infection and the absence of both pericarditis and endocarditis during the active phases of the disease when congestive failure was present. In addition the electrocardiographic changes with grossly abnormal $\mathrm{T}$ waves and low amplitude 
ventricular complexes, and the repeatedly low antistreptolysin titres recorded in the serum are features which are rarely encountered in rheumatic carditis.

Pathology. The constant features seen on histological examination were widespread but patchy necrosis and fragmentation of muscle fibres, and a cellular reaction. Some of these foci of cellular aggregations had an oedematous appearance. The cell type was not constant; neutrophil and eosinophil polymorphonuclear leucocytes, lymphocytes, plasma cells, and macrophages varied in their relative proportions and in one case fibroblast proliferation was present. No correlation was possible between the duration of illness and apparent age of the lesion as judged by the histological pattern. These changes were in no way different from those encountered in myocarditis occurring in infectious diseases.

Kugel and Stoloff (1933) and Kugel (1939) have described a syndrome in infants and young children of cardiac failure due to a non-suppurating myocardial degeneration with cardiac dilatation and hypertrophy. The clinical features of this syndrome closely resemble those of some of our own patients. However, the morbid anatomical changes are different and consist of a very dilated hypertrophied heart with foci of atrophied and degenerated muscle fibres with fibrosis. Occasionally there is a cellular reaction with a few lymphocytes. The relation of these pathological changes to those occurring in idiopathic myocarditis cannot be decided, but they may represent a late stage of idiopathic myocarditis.

Clinical Features. This group of patients demonstrates that idiopathic myocarditis is of variable severity. The lesion may either kill rapidly, resolve completely, or leave residual heart damage. It has been established that myocarditis may cause sudden and unexpected death in infancy (Bowden, 1950), while in others heart failure occurs which may result in death or partial or complete recovery. However, it it is likely that there are mild grades of the disorder which do not cause heart failure and which are unrecognized clinically. Neubauer (1944), Lyon (1947) and others have shown that myocarditis of varying degrees of severity is common in a wide variety of infectious diseases and that death occurs only in the more severe lesions. Pallor, malaise, tachycardia, abnormalities in the quality of the heart sounds and also in the electrocardiogram may be the only manifestations of the disease in the milder cases.

There are two main reasons why myocarditis is rarely diagnosed clinically. First, it is generally believed to be a very uncommon disorder and therefore is rarely considered. Second, the pattern of acute cardiac failure in a young infant can be a confusing one unless encountered previously. Distressed, rapid breathing, cyanosis, cold extremities, poor capillary circulation and tachycardia occur in both cardiac failure and severe acute respiratory tract infections, especially pneumonia. Failure to demonstrate cardiac enlargement is the most important reason for mistaking acute heart failure for pneumonia. It is often impossible in a restless infant with a respiratory rate of 50 to 100 per minute to determine the size of the heart clinically, and in such a patient radiological examination is necessary. Other features such as low or normal temperature with tachycardia, poor quality heart sounds and gallop rhythm, and especially a liver edge palpable three to four fingerbreadths below the costal margin, point to cardiac failure and not pneumonia. It is to be remembered that acute severe bronchiolitis with obstructive emphysema may cause rapid breathing, cyanosis and downward displacement of the liver. The displaced liver in such a patient may easily be mistaken for an enlarged liver.

Paroxysmal tachycardia often gives rise to heart failure if the heart rate is very rapid, and the attack is of some days or weeks' duration. The chief features differentiating the two conditions are the heart rate and alteration in the ventricular complexes and $T$ waves. In myocarditis the rate is rarely above 160 to 170 per minute, while in paroxysmal tachycardia with cardiac failure in infants it is rarely below 200 per minute (Nadas, Daeschner, Roth and Blumenthal, 1952). An electrocardiogram will usually establish the diagnosis but it must be remembered that Lind and Hultquist (1949) have shown that myocarditis may precipitate an attack of paroxysmal tachycardia:

Respiratory distress, cyanosis, and cardiac enlargement in a very young infant may be attributed to heart failure due to a congenital malformation of the heart. The heart faihure may have been precipitated by a respiratory tract infection. Differentiation from heart failure due to myocarditis may be difficult but the following points help to establish the diagnosis. The infant with malformation of the heart usually does not thrive, often has mild attacks of cyanosis, and may be found to have an enlarged heart and murmur some time before cardiac failure develops. In contrast to myocarditis pulmonary engorgement is usually gross, the liver not as large, the heart sounds usually louder and often a murmur is present. Gallop rhythm is rarely heard and the electrocardiographic changes rarely show evidence of gross myocardial damage.

Treatment. Infants with cardiac failure from myocarditis are dangerously ill and gentle nursing and minimal handling are essential. No attempt 
should be made to feed them until congestive failure is relieved and they should be left undisturbed in a warm cot or bassinette in an atmosphere of oxygen. Even minimal handling may lessen the chance of recovery, therefore cooperation and understanding by the nurse in charge of the infant is essential.

The patient should be rapidly brought under control with digitalis. Two patients in the acute group and all in the subacute group responded promptly to the drug so that within 24 hours there was very appreciable improvement, and by 72 hours most of the signs of failure had disappeared. The dose used has been grain $\frac{1}{4}$ to grain $\frac{1}{5}$ of digitalis folium per pound of body weight, or 0.04 of a milligramme of digoxin per pound of body weight over a period of 24 hours. One-third of the total dose may be given immediately and the remainder in three evenly divided doses, at six-hourly intervals. While a few patients were treated satisfactorily with digitalis by mouth during the first 24 hours, it is wiser to use digoxin intramuscularly to obtain a more rapid effect. Oral therapy seems quite satisfactory after the first 24 hours. Digitalis should be continued with a maintenance dose of approximately grain $\frac{1}{40}$ of digitalis folium per pound of body weight until the heart has returned to a normal size or the cardiac lesion healed. It is difficult to decide when healing has taken place, and as cardiac failure in all patients in the subacute group recurred after cessation of several weeks' treatment, it seems advisable to continue digitalis for a minimum period of three months or longer.

Antibiotic drugs were administered to some patients. There was no evidence that either penicillin alone or in combination with streptomycin or aureomycin alone altered the course of the illness.

Prognosis. The prognosis for the infant or child with a short history and severe circulatory failure was poor. The majority did not respond to digitalis therapy and were dead in less than 24 hours from the beginning of treatment. However, two seriously ill patients recovered completely and it is possible that earlier diagnosis and treatment may aid in the recovery of more patients in this group. All patients with the subacute type of illness made a satisfactory initial response to digitalis, four of them survived but have enlarged hearts and two have a mitral valve lesion. The development of a valve lesion was not surprising as one patient who died had infiltration of the atrioventricular ring and the base of the mitral valve with inflammatory cells. The two patients with a valve lesion have been observed for over two and three years respectively, both are in good health and the cardiac lesion seems stationary. Observation over years will be necessary to learn what will happen to these patients. It is possible that young adults with cardiac enlargement of unknown aetiology and with varying degrees of impaired cardiac function developed their disease in infancy or early childhood, and that it is similar in type to the disease in these patients.

\section{Summary}

Idiopathic myocarditis is a serious, frequently fatal malady occurring usually in the first two years of life.

The pathological lesion consists of widespread but usually patchy muscle damage with an associated inflammatory cell reaction.

The common clinical illness is a rapidly developing, usually fatal cardiac failure which is manifested by severe respiratory embarrassment, cyanosis. tachycardia with low or normal temperature, an enlarged heart and liver, and electrocardiographic changes of myocardial damage.

The illness in some patients may be more insidious in onset and the heart failure not as severe. Recovery frequently occurs after a long illness but there is permanent myocardial damage.

The acute form of the disorder is commonly mistaken for pneumonia as respiratory embarrassment and cyanosis are the outstanding clinical features, and cardiac enlargement is difficult and frequently impossible to detect clinically.

Treatment with digitalis and oxygen has been of very definite value in some patients.

The clinical and pathological evidence gives no clue as to the aetiology.

We wish to thank Dr. I. Epstein and Dr. C. Sawrey of the Hamilton Base Hospital for permission to publish cases Nos. 3 and 5, and the Honorary Medical Staff of the Children's Hospital for cases 4, 7, 9, 12 and 13. Dr. H. G. Hiller has kindly reported on the electrocardiograms. Mr. Murphy, Photographic Department, Children's Hospital and Mr. Matthie, Melbourne University, are responsible for the photographs.

\section{REFERENCES}

Bowden, K. M. (1950). Med. J. Aust.. 2. 397.

Gore, I. and Saphir, O. (1947). Amer. Heart J., 34, 827.

Greenebaum, J. B., Felson, W. and Zeligs, M. (1941). J. Pediat., 18, 799.

Keller, H. (1945). Helv. paediat. Acta, 1. 57.

Kenny, F. E. and Sanes, S. (1933). J. Pediat., 3, 321

Kugel, M. A. (1939). Amer. Heart J., 17. 602.

- and Stoloff, E. G. (1933). Amer. J. Dis. Child., 45. 829.

Lind, J. and Hultquist, G. T. (1949). Amer. Heart. J., 38. 123.

Lyon, E. (1947). Med. Rec., N.Y. 160, 402

Maslow, H. L. and Lederer, M. (1933). Amer. J. Dis. Child., 45. 807.

Nadas, A. S., Dauschner, C. W., Roth, A. and Blumenthal, S. L. (1952). Pediatrics, 9. 167.

Neubauer, C. (1944). Archives of Disease in Childhood, 19. 178.

Saphir, O. (1941). Arch. Path., Chicago, 32. 1000.

- (1942). Ibid., 33, 88.

_ and Wile, S. A. (1942). Amer. J. med. Sci., 203, 781.

and Reingold, I. M. (1944). Amer. J. Dis. Child., 67, 294. Scott, R. W. and Saphir, O. (1929). Amer. Heart J., 5, 129. Smith, F. M. and Stephens, R. L. (1938). Trans. Ass. Amer. Phys., 